\title{
MAGNETO-OPTIC PROPERTIES AND OXIDATION/REDUCTION OF ULTRATHIN MAGNETIC FILMS: FE FILMS ON Pt(111)
}

\author{
T.-U. Nahm* \\ Department of Physics and Quantum Photonic Science Research Center, \\ Hanyang University, Seoul 133-791, Korea
}

Received 27 November 2006

\begin{abstract}
We have investigated magneto-optic properties of ultrathin Fe films grown on $\mathrm{Pt}(111)$ surfaces by using the in situ surface magneto-optic Kerr effect (SMOKE) and X-ray photoelectron spectroscopy (XPS). SMOKE measurements show that the Fe layers are not ferromagnetic when the film is thinner than approximately 4.5 MLs (monolayers), but the in-plane magnetization is present for a 4.1 ML Fe film on $\mathrm{Pt}(111)$ annealed at $550 \mathrm{~K}$. Upon post-annealing at $770 \mathrm{~K}$, a 9.2 ML Fe film does not show any Kerr signal, while a 6.3 ML Fe film has the in-plane Kerr signal with increased coercivity. The oxidation and reduction of ultrathin Fe films have also been studied by using XPS. Upon an oxygen exposure of 300 Langmuir at a film temperature of $873 \mathrm{~K}$, the $\mathrm{Fe}$ layers were mostly oxidized as $\mathrm{Fe}_{3} \mathrm{O}_{4}$. When the Fe films were exposed to the same amount of oxygen at room temperature, a partial oxidation as $\mathrm{Fe}_{3} \mathrm{O}_{4}$ was observed for a $3 \mathrm{ML}$ Fe film, while there was no oxidation for a $2 \mathrm{ML} F$ film. On heating the $873 \mathrm{~K}$ oxidized films, $\mathrm{Fe}_{3} \mathrm{O}_{4}$ was reduced to $\mathrm{FeO}$, and even the decomposition was observed. Underlying reasons for these chemical changes of Fe and iron-oxide films are discussed.
\end{abstract}

PACS. 78.20.Ls - 79.60.Jv - 79.60.-i

Keywords: Magneto-optics, photoelectron, magnetic anisotropy, multilayer

\section{INTRODUCTION}

Ultrathin magnetic films have been extensively investigated because of their relation to magnetic alloys and multilayers. The magnetic properties of $\mathrm{Fe} / \mathrm{Pt}$ alloy films and multilayer films have attracted significant attention both for their potential applications as magnetic recording materials and for their scientific interest as a new artificial structure. Extensive studies on these systems have concentrated either on the investigation of the presence of perpendicular magnetic anisotropy (PMA) and its relation to the structure or on the investigation of the local magnetic moments; PMA was observed for Fe/Pt multilayers grown in the (111) direction when $\mathrm{Pt}$ interlayer is thick enough to ensure the low-dimensionality of Fe layers [1], and $\mathrm{Fe} / \mathrm{Pt}$ multilayers grown in the (100) direction in certain cases [2, 3]. It is also reported that Pt atoms in $\mathrm{Fe} / \mathrm{Pt}$ multilayers and $\mathrm{Fe}-\mathrm{Pt}$ alloys have induced magnetic moments [4].

In relation to these novel properties of Fe-Pt alloy films and multilayers, it is necessary to study the role of interface and intermixing between the ad-layer and the substrate. We have previously studied ultrathin Fe films on Pd(111) [5], and found that the morphology of the interface and the

\footnotetext{
* Corresponding author e-mail: tschnahm@ hanyang.ac.kr
} 
degree of intermixing is very crucial to the magnetic properties of ultrathin films. Our preliminary results on Fe films on $\mathrm{Pt}(111)$ [6] also confirmed the importance of interdiffusion between the adatoms and the substrate.

In addition to the interest in magnetic recording materials, spin-dependent electron transport in magnetic tunnel junctions (MTJs) has been studied intensively for such attractive applications as magnetic sensors and nonvolatile magnetic memory devices. Since the tunnel magnetoresis-tance of an MTJ depends on the spin polarization of the ferromagnetic electrodes, it is ideal to use materials with $100 \%$ polarization at the Fermi level which can in principle give an infinite value of MR. The technological importance and scientific interest in half-metallicity lead to a great number of studies on the structure and magnetic properties of half-metallic ferromagnet films, and many theoretical studies predict half-metallicity of $\mathrm{CrO}_{2}$ [7] and magnetite $\left(\mathrm{Fe}_{3} \mathrm{O}_{4}\right)[8]$.

Epitaxial $\mathrm{Fe}_{3} \mathrm{O}_{4}$ films have been grown on $\mathrm{W}(110)$ [9], $\mathrm{MgO}(100)$ [10], $\mathrm{MgO}(111)$ [11], and $\operatorname{Pt}(111)$ [12], and some reports on spin polarization have been made [13, 14]. However, detailed studies on the effect of annealing of the ultrathin $\mathrm{Fe}_{3} \mathrm{O}_{4}$ films has not been performed.

In this work, we have investigated Fe films grown on $\mathrm{Pt}(111)$ by using the surface magnetooptic Kerr effect (SMOKE) and X-ray photoelectron spectroscopy (XPS). We found that the Fe layers are not ferromagnetic when the film is thinner than 4.5 MLs (monolayers), and that the in-plane magnetization is present for a $4.1 \mathrm{ML}$ upon post-annealing at $550 \mathrm{~K}$. Thicker films show only in-plane Kerr signal. Upon post-annealing at $770 \mathrm{~K}$, a 9.2 ML Fe film does not show any Kerr signal, while a 6.3 ML Fe film has in-plane Kerr signal with increased coercivity, and the average compositions of the intermixed layers caused by the interdiffusion of $\mathrm{Fe}$ atoms into the Pt substrate were also estimated. We also grew iron oxide films on $\mathrm{Pt}(111)$ substrates by oxidizing 2 or $3 \mathrm{ML} \mathrm{Fe}$ films at $873 \mathrm{~K}$ and at room temperature. In order to determine the oxidation state of the oxidized film, we used X-ray photoelectron spectroscopy. It was found that upon an oxygen exposure of 300 Langmuir and at film temperature of $873 \mathrm{~K}$, both layers are oxidized as $\mathrm{Fe}_{3} \mathrm{O}_{4}$, but only partial or no oxidation was observed when the film was kept at room temperature. On heating the $873 \mathrm{~K}$ oxidized films, $\mathrm{Fe}_{3} \mathrm{O}_{4}$ layer was reduced to $\mathrm{FeO}$, and even decomposition was observed.

\section{EXPERIMENTS}

The SMOKE and XPS measurements were performed in a home-made ultrahigh vacuum chamber. The base pressure of the chamber was $2 \times 10^{-10}$ torr. The $\operatorname{Pt}(111)$ substrate was of the disk type with a $10 \mathrm{~mm}$ diameter and $1 \mathrm{~mm}$ thickness. The surface was cleaned by several cycles of $\mathrm{Ar}^{+}$sputtering at $1 \mathrm{keV}$ and annealing at $870 \mathrm{~K}$ in the UHV chamber until a sharp $(1 \times$ 1) low energy electron diffraction (LEED) pattern was observed. No contamination was detected in the XPS spectrum. The XPS spectra were obtained with unmonochromatized Al Ka radiation with $\mathrm{h} v=1486.6 \mathrm{eV}$. The overall resolution was $0.9 \mathrm{eV}$. The light source for SMOKE measurement was a $10 \mathrm{~mW} \mathrm{He}-\mathrm{Ne}$ laser with $\lambda=623.8 \mathrm{~nm}$.

The Fe film was deposited on the substrate at room temperature (RT) by an e-beam heating method. An iron wire of $99.99 \%$ purity was heated by electron bombardment from a tungsten filament. The film thickness was first calibrated by using a quartz thickness monitor, and was then cross-checked by XPS by using $F e 2 p$ and Pt4f core-level photoelectron (PE) intensities at sub-ML coverage, assuming no island growth. In this work, the Fe film thickness was represented in units of one monolayer and $1 \mathrm{ML}$ was set as $1.50 \times 10^{15} \mathrm{Fe}$ atoms $\mathrm{cm}^{-2}$, assuming a pseu-domorphic growth of the Fe film on the $\mathrm{Pt}(111)$ surface. Most post-annealing was done for $3 \mathrm{~min}$ at a given temperature, unless otherwise stated. For oxidation/reduction study, the $\mathrm{Fe}$ films were oxidized by backfilling of $\mathrm{O}_{2}$ for $5 \mathrm{~min}$ with a partial pressure of $1.0 \times 10^{-6}$ Torr, when the film was kept either at room temperature or at $873 \mathrm{~K}$. Post-annealing of Fe-oxide films

108 
was performed for $5 \mathrm{~min}$ at various temperatures.

\section{RESULTS AND DISCUSSION}

\subsection{Ultrathin Fe films on Pt(111)}

The results of SMOKE measurement for a 6.3 ML Fe film grown on Pt(111) surface are shown in Fig. 1. The Kerr rotation of a 6.3 ML Fe film has a well-defined in-plane hysteresis curve with a low coercivity, and post-annealing at $640 \mathrm{~K}$ does not change it much. However, the coercivity increases to 110 Oe upon further annealing at $740 \mathrm{~K}$. This increase was also observed for Fe films on $\mathrm{Pd}(111)$ at $\mathrm{T}=600-660 \mathrm{~K}$ [5]. On the other hand, no hysteresis curves were observed for films with Fe thickness less than 4.5 MLs. The as-deposited 4.1 ML Fe film does not show Kerr signal for any direction, but upon annealing at $550 \mathrm{~K}$ for $15 \mathrm{~min}$, the in-plane Kerr signal is obtained with a coercivity of 2.9 Oe [6].

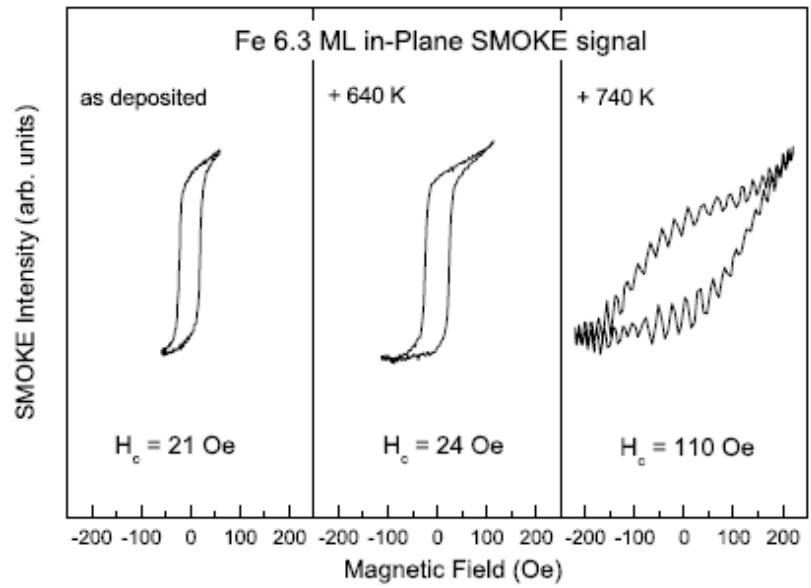

Fig. 1: Kerr signals from 6.3 ML Fe films on Pt(111) after RT deposition and upon annealing at given temperatures

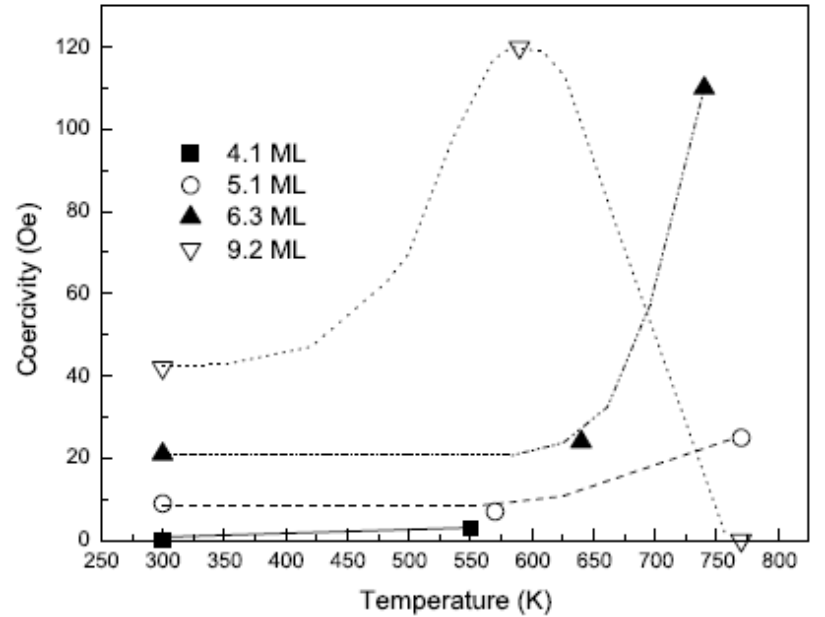

Fig. 2: Coercivity values of 4.1, 5.1, 6.3, and 9.2 ML Fe films on Pt(111) upon post-annealing. The lines are guides to an eye 
Figure 2 shows the change in coercivity of various films upon annealing. For 4.1 and 5.1 ML Fe films, we observed an increase of the coercivity as reported in Ref. [6]. Since the coercivity tends to vanish as the temperature approaches Tc due to a rapid decrease in all contributions to the magnetic anisotropy [15], the increase in coercivity upon annealing may imply that the film has higher Tc than the as-deposited case, when we assume that there is little change in grain size. Although bulk Fe-Pt alloys have lower Tc than bulk Fe, it is also well known that Tc and co-ercivity are dependent upon thickness. Since the thickness of intermixed layers must be larger than that of the as-deposited Fe layers, the Tc of some intermixed layers can be higher than that of the as-deposited Fe layers. This explains the appearance of hysteresis curve upon annealing of 4.1 ML Fe film. A formation of compositionally inho-mogeneous layers may results in a reduction of the size of magnetic domains, which can also give a larger value of coercivity, and we conjecture that both of the two are the primary factors of the observed increase in coercivity.

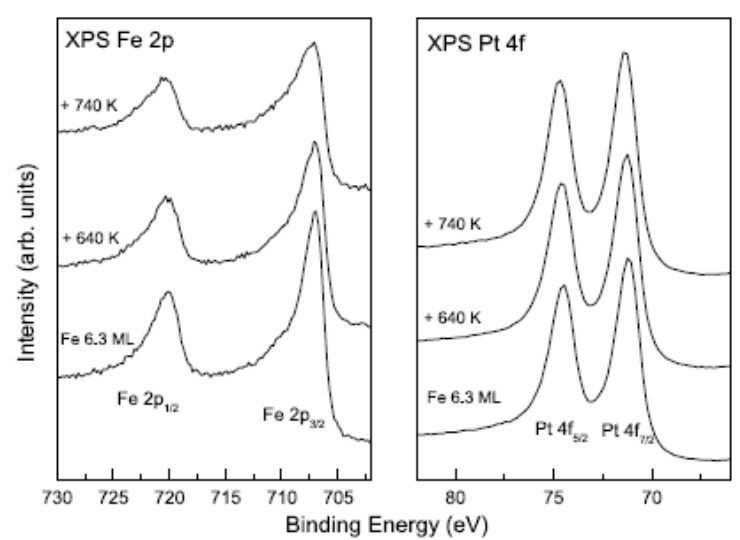

Fig. 3: XPS spectra of a $6.3 \mathrm{ML}$ Fe films on Pt(111) after RT deposition, upon $640 \mathrm{~K}$ and $740 \mathrm{~K}$ post-annealing

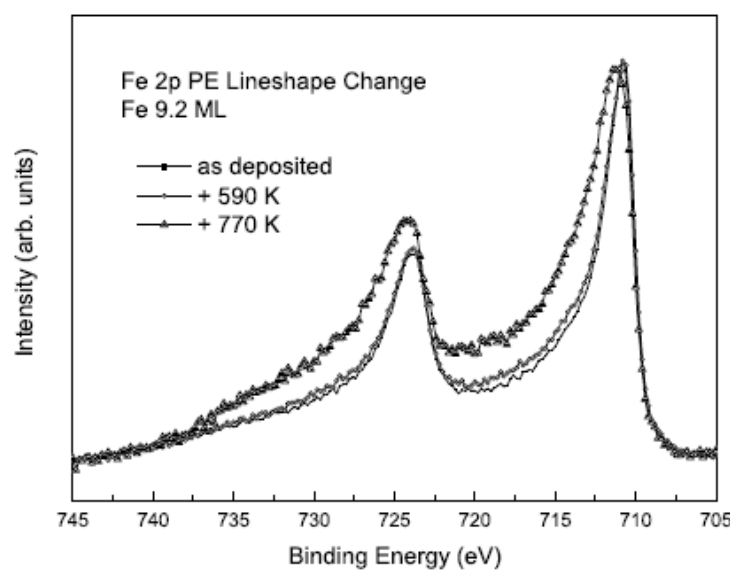

Fig. 4: Fe2p core-level spectra of 9.2 ML Fe films on Pt(111), as-deposition, after annealing at $590 \mathrm{~K}$, and after annealing at $770 \mathrm{~K}$

As we reported in Ref. [6], however, post-annealing of 9.2 ML Fe film at $770 \mathrm{~K}$ results in the disappearance of hysteresis curve. We also measured the Kerr ellipticity, but found no signal. In a preliminary study, we speculated that the absence of hysteresis curve for a $9.2 \mathrm{ML} \mathrm{Fe}$ film 110 
annealed at $770 \mathrm{~K}$ is caused by the tetragonal distortion and $\mathrm{Fe}_{50} \mathrm{Pt}_{50}$ surface alloy formation upon annealing [6]; it is known that there is a competition between ferromag-netism and antiferromagnetism in fct $\mathrm{FePt}$ [16].

Figure 3 represents the core-level spectra of Pt4f and Fe $2 p$ PE lines from 6.3 ML Fe film on $\mathrm{Pt}(111)$. The $F e 2 p \mathrm{PE}$ line does not change its lineshape greatly upon annealing, which is quite different from 9.2 ML Fe film (Fig. 4). Since the $F e 2 p$ PE spectra have additional satellite structures at higher-binding side of the main peak due to many-body effect when $\mathrm{Fe}$ is alloyed with noble metals, Pd, and Pt [5, 17], the average Fe content of the intermixed layers of postannealed 9.2 ML Fe film must be smaller than that of 6.3 ML film. Since the change in lineshape is not so severe as in $\mathrm{Fe} / \mathrm{Pd}(111)$ case [5], we can assume that the average compositions of intermixed layers of both cases are different from the post-annealed $\mathrm{Fe} / \mathrm{Pd}(111)$, where the composition of thick layer was estimated to be in between 9 and 15 at.\% Fe.

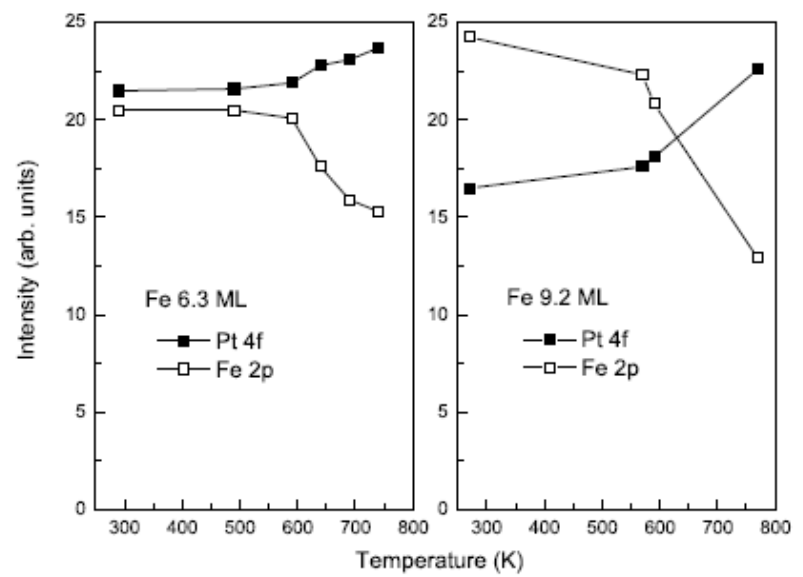

Fig. 5: Photoelectron intensity change from 6.3 and 9.2 ML Fe films on Pt(111) as a function of post-annealing temperature. (Right panel of the figure is from Ref. [6])

As in Ref. [6], when we compare the PE intensity ratio of RT and $770 \mathrm{~K}$ annealed films, we can get rough estimate of the average Fe concentration, since the pho-toelectrons emitted from the inner layers decay through inelastic scatterings [18]. Figure 5 shows the changes in the PE intensities from 6.3 and 9.2 ML Fe films upon annealing at given temperatures. Assuming a pseudomorphic behavior for the as-deposited 6.3 ML Fe film, the PE intensity ratio is given by

$$
\frac{I_{F e 2 p}}{I_{P t 4 f}}=A \frac{0.7 \sum_{n=0}^{5} \exp \left(-\frac{n d}{\lambda_{F e}}\right)+0.3 \sum_{n=0}^{6} \exp \left(-\frac{n d}{\lambda_{F e}}\right)}{0.7 \sum_{n=6}^{\infty} \exp \left(-\frac{n d}{\lambda_{P t}}\right)+0.3 \sum_{n=7}^{\infty} \exp \left(-\frac{n d}{\lambda_{P t}}\right)},
$$

where $\mathrm{d}$ is the interlayer spacing of $\operatorname{Pt}(111)$. The electron inelastic mean free paths have the values $\lambda_{F e}=5.44 \mathrm{ML}$ and $\lambda_{P t}=7.33 \mathrm{ML}$ for $\mathrm{Fe} 2 \mathrm{p}$ and $P t 4 f$ photoelectrons excited by $\mathrm{Al} \mathrm{K \alpha}$ radiation [18]. A constant $A$ represents the ratio of the photoionization cross sections and the effect of analyzer transmission function. If we assume that the surface alloy forms homogeneously with sharp interface upon $740 \mathrm{~K}$ annealing, the PE intensity ratio would be

$$
\frac{I_{F e 2 p}}{I_{P t 4 f}}=A \frac{x \sum_{n=0}^{l-1} \exp \left(-\frac{n d}{\lambda_{F e}}\right)}{(1-x) \sum_{n=0}^{l-1} \exp \left(-\frac{n d}{\lambda_{P t}}\right)+\sum_{n=l}^{\infty} \exp \left(-\frac{n d}{\lambda_{P t}}\right)},
$$


ith the constraint

$$
x \times l=6.3,
$$

where $x$ is the Fe concentration and 1 is the thickness of surface alloy in monolayers. This gives $x=0.68$, which is larger than the value for the $9.2 \mathrm{ML}$ Fe film after annealing at $770 \mathrm{~K}, 0.49$. This is consistent with the lineshape change in the $F e 2 p$ core-level spectra of the $9.2 \mathrm{ML} \mathrm{Fe}$ film.

The homogeneity of the intermixed layers can be estimated from the change in linewidth of the PE lines. It is well known that Pt4f lineshape does not follow usual Doniach-Sunjic type, but Mahan type, and it is not easy to determine the real half-width when asymmetry is not treated rigorously [19]. Here we fitted only part of PE lines where simple Voigt function worked. Binding energy shifts and the linewidth change of Pt4f PE lines from the fitting are shown in Fig. 6. The binding energy shift is resulted from the intermixing of Pt substrate with $\mathrm{Fe}$ atoms and its maximum value is 0.17 and $0.25 \mathrm{eV}$ for 6.3 and 9.2 ML Fe films, respectively. The values of full-width at half-maximum (FWHM) of Pt4f PE lines is decreased upon deposition of Fe atoms at RT. This might be related to the change of surface states. The value of FWHM is increased upon annealing at 570 and $590 \mathrm{~K}$, indicating that the Pt atoms have many different chemical environments. This can be understood from the normal diffusion phenomena. However, upon annealing the 9.2 ML Fe film at $770 \mathrm{~K}$, the value of FWHM is decreased from $1.39 \mathrm{eV}$ to $1.27 \mathrm{eV}$, and we can conclude that the intermixed layers have more or less homogeneous chemical environments, unlike the 6.3 ML Fe film. From the results, it is clear that the $770 \mathrm{~K}$ annealed intermixed layer of the 9.2 $\mathrm{ML} \mathrm{Fe}$ film is composed of nearly equiatomic amount of $\mathrm{Fe}$ and $\mathrm{Pt}$ atoms.

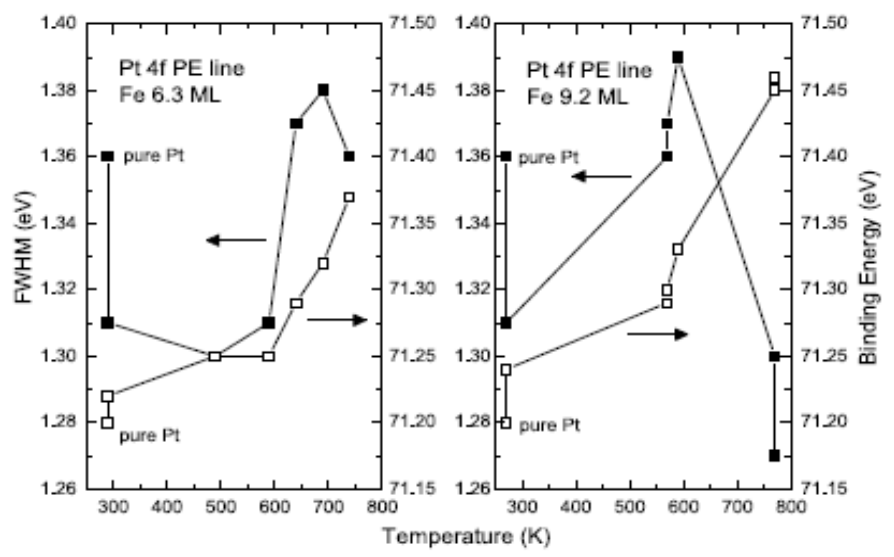

Fig. 6: Binding energy shift (open squares) and full-width at half-maximum (solid squares) of Pt4f PE lines as a function of post-annealing temperature. The values of pure Pt(111) is shown for comparison. (Right panel of the figure is from Ref. [6])

Figure 7 represents the valence band PE spectra from 6.3 and 9.2 ML Fe films. Since the values of the photoionization cross sections of Pt5d and Fe3d states with Al Ka radiation are 0.021 and $0.0022 \mathrm{Mb}$ [20], the PE spectra are predominantly determined by the Pt partial spectral weights. Therefore, there are little change in the spectra even when 9.2 ML of Fe atoms are deposited on $\operatorname{Pt}(111)$ substrate. However, upon annealing at high temperatures, the spectra lose their weights near the Fermi level, as indicated by arrows in Fig. 7. It is apparent that the change of the lineshape is more evident in the spectra of the 9.2 ML Fe film taken after annealing at $770 \mathrm{~K}$. This is also in accord with all our observation that the Fe atoms in 9.2 ML Fe film interdiffuse into the substrate more than those in 6.3 ML Fe film. Actually, the spectra of the 9.2 ML Fe film 
taken after annealing at $770 \mathrm{~K}$ is quite similar to the Pt5d partial spectral weights of $\mathrm{Fe}_{50} \mathrm{Pt}_{50}$, and that of the 6.3 ML Fe film taken after annealing at $740 \mathrm{~K}$ has a shape close to the partial spectral weights of $\mathrm{Fe}_{75} \mathrm{Pt}_{25}$ determined by using synchrotron radiation [21]. The difference in the overall shapes between the current X-ray PE spectra and the partial spectral weights in Ref. $[21]$ is due to the matrix element effect in photoionization process.

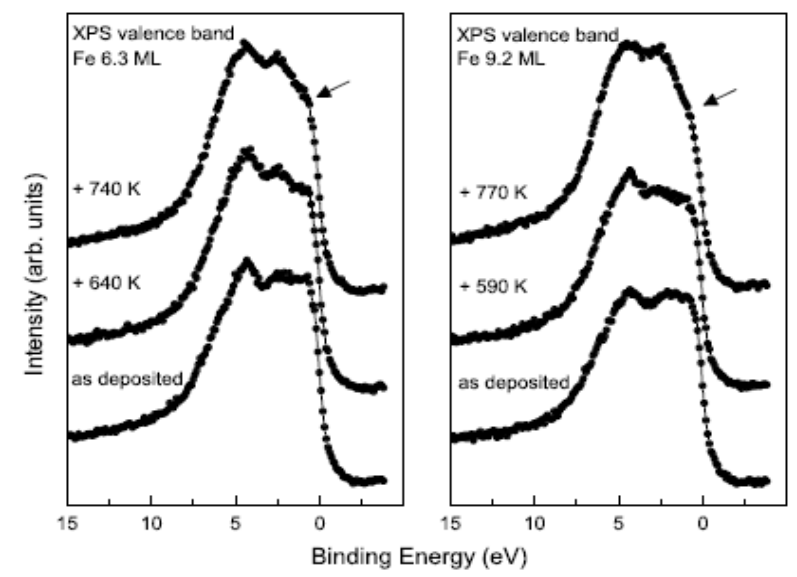

Fig. 7: Kerr signals from 6.3 ML Fe films on Pt(111) after RT deposition and upon annealing at given temperatures

\subsection{Oxidation and reduction}

Figure 8 shows the $\mathrm{Fe} 2 p$ core level PE spectra of the oxidized 3 and 2 ML Fe films on Pt(111) before and after post-annealing at $961 \mathrm{~K}$. The film temperature during an oxygen exposure of 300 Langmuir was $873 \mathrm{~K}$. These spectra are quite complex relative to that of Fe metal due to the presence of shake-up satellites and multiplet splittings in the $\mathrm{Fe} 2 p$ PE final states in Fe oxides [22]. The spectra for $\mathrm{Fe}_{3} \mathrm{O}_{4}$ are even more complicated by the presence of two oxidation states of Fe.

Upon post-annealing the films, we observed a big difference between the two films. As can be seen in Fig. 8, the peak position of the $\mathrm{Fe} 2 p_{3 / 2}$ PE line shifts towards lower binding-energy side in both films. The change of the binding energy indicates that the oxide films, mostly of the $\mathrm{Fe}_{3} \mathrm{O}_{4}$ phase, contain mixed phases of $\mathrm{Fe}_{3} \mathrm{O}_{4}$ and $\mathrm{FeO}$ upon low temperature annealing [23]. High temperature annealing of films leads to a decrease in the relative amount of $\mathrm{Fe}^{3+}$. While the final phase of the oxidized $3 \mathrm{ML}$ Fe film upon post-annealing is dominantly $\mathrm{FeO}$, the $2 \mathrm{ML}$ case is quite different; the oxides are even decomposed.

The reduction and decomposition of Fe oxides are not thermodynamically favorable, when they are in bulk state. All Fe oxides have negative values of heat of formation at room temperature ($11.6 \mathrm{eV} /$ molecule for $\mathrm{Fe}_{3} \mathrm{O}_{4},-8.53$ for $\mathrm{Fe}_{2} \mathrm{O}_{3}$, and -2.75 for $\mathrm{FeO}$ [24]), and it is hardly conceivable that decomposition can occur even without being evaporated as clusters. However, if the oxides are in thin films, the interaction with the substrate can enhance reduction by several ways. If the substrate metal has a large negative value of heat of formation for oxidation, reduction and decomposition of oxide film can occur; this is energetically favorable, and heat energy can be used to overcome the activation energy barrier [25]. However, $\operatorname{Pt}(111)$ surface is very inert to the oxidation and the platinum oxide, $\mathrm{PtO}_{2}$, has a positive value of heat of formation [26]. Another possible way is the intermixing of metallic ad-atoms and substrate atoms; if the partial heat of formation of diluted alloy with the ad-atoms as impurity has a large negative value, then this process is also energetically favorable. Since Fe-Pt alloys form a 
continuous solid solution, the latter is probably the cause of the observed reduction and decomposition, and the whole process can be summarized as

$$
\begin{aligned}
& 2 \mathrm{Fe}_{3} \mathrm{O}_{4}+\mathrm{Pt} \text { substrate } \\
\rightarrow & 6 \mathrm{FeO}+\mathrm{O}_{2}+\mathrm{Pt} \text { substrate }
\end{aligned}
$$

$\rightarrow \mathrm{Fe}-\mathrm{Pt}$ alloy (interface) $+4 \mathrm{O}_{2}$.

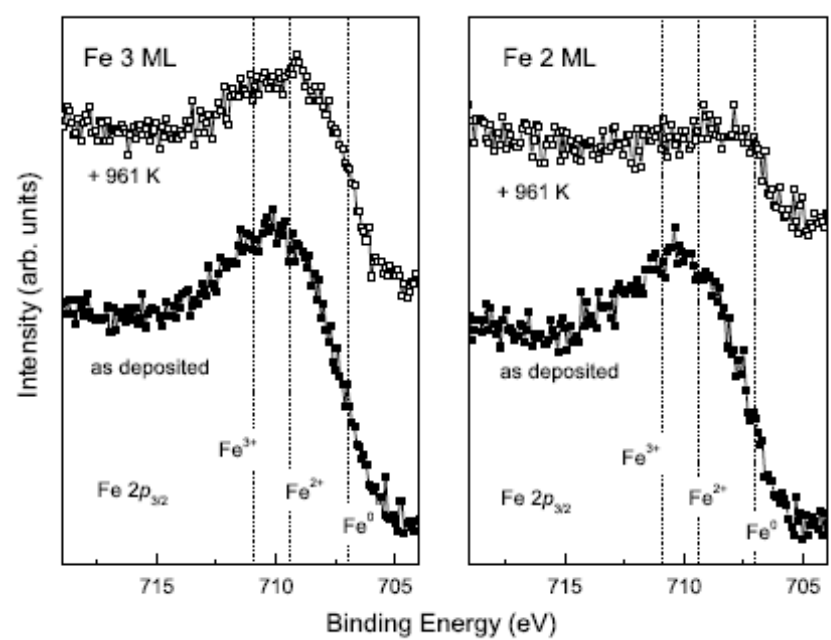

Fig. 8: Fe2p spectra of the 3 (left panel) and 2 ML (right panel) Fe films on Pt(111): after 5 min oxidation at $873 \mathrm{~K}$ (bottom) and after annealing for $10 \mathrm{~min}$ at $961 \mathrm{~K}$. The vertical lines indicates the peak position of $\mathrm{Fe}_{2} \mathrm{O}_{3}\left(\mathrm{Fe}^{3+}\right)$. $\mathrm{FeO}\left(\mathrm{Fe}^{2+}\right)$, and metallic Fe phases. All the data from Ref. [23]

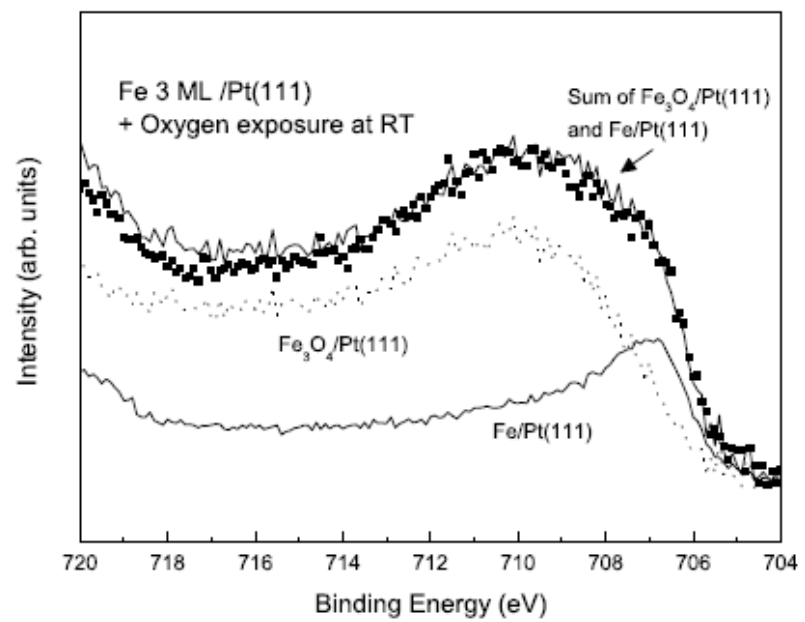

Fig. 9: Fe2p spectra of the 3 ML Fe films on Pt(111) after 5 min oxidation at RT (squares) fitted with the spectra of fully oxidized films (dots) and that of as-deposited film (thin solid line). The solid line is the fitting result

Figure 9 shows the $F e 2 p$ core level photoemission(PE) spectra of the oxidized 3 ML Fe films on $\mathrm{Pt}(111)$. The film was kept at room temperautre during an oxygen exposure of 300 Langmuir. It is apparent that the $\mathrm{Fe} 2 p$ spectrum of as-oxidized $3 \mathrm{ML} \mathrm{Fe}$ film is a composite of the two 114 
spectra, $\mathrm{Fe} 2 p$ of $\mathrm{Fe}_{3} \mathrm{O}_{4}$ and that of metallic $\mathrm{Fe}$. Probably only the top layers are oxidized, and the Fe layers near the interface seems to be in its metallic phase.

In order to estimate the amount of Fe atoms still in its metallic phase, we tried to fit the spectra combining the $F e 2 p$ spectrum of the as-deposited $3 \mathrm{ML} \mathrm{Fe}$ film and that of the $3 \mathrm{ML} F$ oxidized at $873 \mathrm{~K}$, assuming that the latter is similar to the spectrum of bulk $\mathrm{Fe}_{3} \mathrm{O}_{4}$. The best fit can be achieved by combining $1 / 5$ of the spectrum of the as-deposited and 4/5 of the spectrum of the fully oxidized $3 \mathrm{ML}$ Fe film. When we use a similar method as in eqs. (1) and (2) by assuming that the top layers are oxides and that the metallic $\mathrm{Fe}$ atoms are in between the oxide layers and the substrate,

and

$$
\frac{1}{5}=\exp \left(-\frac{n_{\text {oxide }} d_{\text {oxide }}}{\lambda_{\text {Feloxide }}}\right) \frac{1-\exp \left(-\frac{n_{F e} d_{F e}}{\lambda_{F e / F e}}\right)}{1-\exp \left(-\frac{3 d_{F e}}{\lambda_{F e / F e}}\right)}
$$

$$
\frac{4}{5}=\frac{1-\exp \left(-\frac{n_{\text {oxide }} d_{\text {oxide }}}{\lambda_{\text {Feloxide }}}\right)}{1-\exp \left(-\frac{4.95 d_{F e}}{\lambda_{\text {Feloxide }}}\right)}
$$

where $d_{F e}=2.26 \mathrm{~A}$ and $d_{\text {oxide }}=2.20 \mathrm{~A}$ are the interlayer spacing of Fe layers on $\mathrm{Pt}(111)$ and that of $\mathrm{Fe}_{3} \mathrm{O}_{4}$ layers as defined in Ref. [18], and $n_{F e}$ and noxide are the number of Fe layers and that of $\mathrm{Fe}_{3} \mathrm{O}_{4}$ layers, respectively. The electron inelastic mean free paths in $\mathrm{Fe}$ and in $\mathrm{Fe}_{3} \mathrm{O}_{4}$ have the values $\lambda_{F e / F e}=5.44 \mathrm{ML}$ and $\lambda_{\text {Fe/oxide }}=9.53 \mathrm{ML}$ for $\mathrm{Fe} 2 p$ photoelectrons excited by $\mathrm{Al} \mathrm{K \alpha}$ radiation [18]. Then eqs. (3) and (4) give $n_{F e}=0.73$ and $n_{\text {oxide }}=3.73$. Considering that the thickness of $\mathrm{a} \mathrm{Fe}_{3} \mathrm{O}_{4}$ film is 1.65 times that of the $\mathrm{Fe}$ film with the same amount of $\mathrm{Fe}$ atoms, we can predict that the amount of Fe atoms in oxide corresponds to 2.26 MLs of Fe on $\mathrm{Pt}(111)$ surface. The sum of the amount of the Fe atoms in oxide phase and in metallic phase thus corresponds to $2.99 \mathrm{MLs}$ of Fe on Pt(111), which is almost exactly what one can expect. Hence we can conclude that the metallic Fe layer in between the oxide layers and the substrate is less than a ML.

We also studied the oxidation of a $2 \mathrm{ML}$ Fe film at room temperature. Different from the $3 \mathrm{ML}$ case, we cannot observe any $\mathrm{Fe}^{2+}$ or $\mathrm{Fe}^{3+}$ peaks, and it can be concluded that the Fe layers are not oxidized with an oxygen exposure of 300 Langmuir. Although a strong hybridization between $\mathrm{Fe} 3 d$ states and Pt5d states may inhibit the oxidation of the first layer, it is not easy to understand why this inhibition lasts up to a film thickness of 2 MLs. We are currently studying the oxidation of the Fe films on $\mathrm{Pt}(111)$ and $\mathrm{Pt}(100)$ with much higher oxygen dose to settle this question.

\section{CONCLUSION}

In summary, the magnetic properties and oxidation of ultrathin Fe films grown on a $\mathrm{Pt}(111)$ surface were investigated by using SMOKE and XPS. By combining the experimental results, it was found that the Fe layers are not ferromagnetic when the film is thinner than approximately 4.5 MLs, but that in-plane magnetization is present for a 4.1 ML Fe film on $\mathrm{Pt}(111)$ upon annealing at $550 \mathrm{~K}$. Upon post-annealing at $770 \mathrm{~K}$, a 9.2 ML Fe film does not show any Kerr signal, while a 6.3 ML Fe film has in-plane Kerr signal with increased coercivity. The average compositions of the intermixed layers caused by the interdiffusion of $\mathrm{Fe}$ atoms into the $\mathrm{Pt}$ substrate are estimated to be 68 and 49 at.\% Fe for 6.3 and 9.2 ML case, respectively. The ab- 
sence of ferromagnetic order observed in a $770 \mathrm{~K}$ annealed 9.2 ML Fe film might be resulted from the tetragonal distortion and chemical ordering. We also found that upon an oxygen exposure of 300 Langmuir at a film temperature of $873 \mathrm{~K}$, the Fe films were oxidized mostly as $\mathrm{Fe}_{3} \mathrm{O}_{4}$. When the Fe films were exposed to the same amount of oxygen at room temperature, partial oxidation as $\mathrm{Fe}_{3} \mathrm{O}_{4}$ was observed for a $3 \mathrm{ML} F$ film, while there was no oxidation for a 2 ML Fe film. On heating the $873 \mathrm{~K}$ oxidized films, $\mathrm{Fe}_{3} \mathrm{O}_{4}$ was reduced to $\mathrm{FeO}$, and even decomposition was observed. Most of these complex behaviors can be understood qualitatively when we consider the changes in thermodynamical quantities during various chemical reactions. The inhibition of the RT oxidization of a $2 \mathrm{ML}$ Fe film is probably due to a rather strong hybridization between $\mathrm{Fe}$ and $\mathrm{Pt} d$ states.

\section{ACKNOWLEDGEMENT}

This work was supported by MOST/KOSEF through the Quantum Photonic Science Research Center.

\section{REFERENCES}

1. Simopoulos, A, Devlin, E., Kostikas, A., Jankowski, A., Croft, M., and Tsakalakos, T. (1996), Phys. Rev., vol. B 54, p. 9931.

2. Katayama, T., Suzuki, Y., Nishihara, Y., Sugimoto, T., and Hashimoto, M. (1991), J. Appl. Phys., vol. 69, p. 5858.

3. Antel, W.J.Jr., Schwickert, M.M., Lin, T., O'Brien, W.L., and Harp, G.R. (1999), Phys. Rev., vol. B 60, p. 12933.

4. Koide, T., Shidara, T., Yamaguchi, K., Fujimori, A., Fuku- tani, H., Nakajima, N., Sugimoto, T., Katayama, T., and Suzuki, Y. (1996), Phys. Rev., vol. B 53, p. 8219.

5. Choi, J.-H., Nahm, T.-U., Kim, W., Kim, W., Chung, J., Kim, J.-Y., Koh, H., and Oh, S.-J. (2001), Surf. Sci., vol. 495, p. 173.

6. Nahm, T.-U., Kim, W., and Oh, S.-J. (2005), J. Korean Phys. Soc., vol. 46, p. S125.

7. Schwarz, K.-H. (1986), J. Phys. F: Met. Phys., vol. 16, p. L211.

8. Zhang, Z. and Satpathy, S. (1991), Phys. Rev.,vol. B 44, p. 13319.

9. Kim, H.-J., Park, J.-H., and Vescovo, E. (2000), Phys. Rev., vol. B 61, p. 15284.

10. Chambers, S.A., Thevuthasan, S., and Joyce, S. (2000), Surf. Sci., vol. 450, p. L273.

11. Lazarov, K., Weinert, M., Chambers, S.A., and Gajdardziska-Josifovska, M. (2005), Phys. Rev., vol. B 72, p. 195401.

12. Weiss, W., Barbieri, A., Van Hove, M.A., and Somorjai, G.A. (1993), Phys. Rev. Lett., vol. 71, p. 1848.

13. Dedkov, Yu.S., Riidige, U., and Giintherodt, G. (2002), Phys. Rev., vol. B 65, p. 064417.

14. Fonin, M., Dedkov, Yu.S., Mayer, J., Riidiger, U., and Giintherodt, G. (2003), Phys. Rev., vol. B 68, p. 045414.

15. Camarero, J., de Miguel, J.J., Miranda, R., and Her-nando, A. (2000), J. Phys.: Condens. Matter, vol. 12, p. 7713.

16. Brown, G., Kraczek, B., Janotti, A., Schulthess, T.C., Stocks, G.M., and Johnson, D.D. (2003), Phys. Rev., vol. B 68, p. 052405. 
17. Nahm, T.-U., Noh, H.-J., Choi, B., Park, J.-S., Oh, S.-J., and Cho, E.-J. (2003), J. Phys.: Condens. Matter, vol. 15, p. 3181.

18. Seah, M.P. and Dench, W. A. (1979), Surf. Interf. Anal., vol. 1, p. 1.

19. Ley, L. and Cardona, M. (eds.) (1979), Photoemission in Solids II (Springer Verlag, Berlin), p. 351.

20. Yeh, J.J. and Lindau, I. (1979), At. Data Nucl. Data Tables, vol. 32, p. 1.

21. Song, S.-H., Park, J.-S., Nahm, T.-U., Noh, H.-J., Choi, B.-H., and Oh, S.-J. (2004), J. Korean Phys. Soc., vol. 45, p. 51.

22. Schedel-Niedrig, Th., Weiss, W., and Schlogl, R. (1995), Phys. Rev., vol. B 52, p. 17449.

23. Byun, B.-S., Kim, M.-S., and Nahm, T.-U. (2006), J. Korean Phys. Soc., vol. 49, p. 1006.

24. Kubaschewski, O. and Alcock, C.B. (1979), Metallurgical Thermochemistry (Pergamon Press, Oxford), p. 284.

25. Nahm, T.-U. and Gomer, R. (1997), Surf. Sci., vol. 373, p. 237.

26. Ref. [24], p. 304. 